\title{
Article
}

\section{Nonparametric Identification for Control of MIMO Hammerstein Systems}

\author{
Jyh-Cheng Jeng, and Hsiao-Ping Huang
}

Ind. Eng. Chem. Res., 2008, 47 (17), 6640-6647 • DOI: 10.1021/ie071512q • Publication Date (Web): 30 July 2008

Downloaded from http://pubs.acs.org on November 18, 2008

\section{More About This Article}

Additional resources and features associated with this article are available within the HTML version:

- Supporting Information

- $\quad$ Access to high resolution figures

- $\quad$ Links to articles and content related to this article

- Copyright permission to reproduce figures and/or text from this article

\section{View the Full Text HTML}




\title{
Nonparametric Identification for Control of MIMO Hammerstein Systems
}

\author{
Jyh-Cheng Jeng and Hsiao-Ping Huang* \\ Department of Chemical Engineering, National Taiwan University, Taipei 106, Taiwan
}

\begin{abstract}
A new nonparametric method to identify multivariable Hammerstein models is presented. The Hammerstein model is characterized by a combination of a linear dynamic subsystem and an algebraic nonlinear function. There could be many different models that give the same input-output realization. The purpose of this identification is to find out one among those models for controller design. This identification uses a sequence of specially designed test signals for excitation. The linear dynamic subsystem is identified as a finite sequence of impulse response (FIR), and the static nonlinearity is identified as a multi-input-multi-output (MIMO) functional mapping. By making use of this special test signal, the FIR sequence can be estimated under a single-input-single-output (SISO) framework. Moreover, the identification for linear subsystem can be decoupled from that for the nonlinear static part. This nonparametric model can be used for model predictive control applications.
\end{abstract}

\section{Introduction}

Most of the dynamics of chemical processes are nonlinear in nature. In order to be able to describe the global behavior of the system, many research activities have been focused on developing methods for modeling and identification of such nonlinear systems. One of the most frequently used nonlinear models is the Hammerstein model, which is composed of a nonmemory function as the static nonlinearity part followed by a linear dynamical subsystem. Because of this model structure, the model expressions of the process are not unique, especially, for multi-input-multi-output (MIMO) systems.

Many methods for modeling and identification of Hammersteintype systems published in the open literature focused on the singleinput-single-output (SISO) systems. Only a few exceptions dealt with the MIMO systems (e.g., Verhaegen and Westwick; ${ }^{1} \mathrm{Al}-$ Duwaish and Karim; ${ }^{2}$ Patwardhan et al. ${ }^{3}$ Rollins et al..; ${ }^{4}$ Lakshminarayanan et al. ${ }^{5}$ ). The methods for identification of MIMO Hammerstein system differ mainly in the way the static nonlinearity is represented and the optimization problem finally formulated. In parametric approaches, the static functional nonlinearity is expressed in terms of polynomials, neural networks, and some expansions of basis functions. A priori process knowledge is required to select an appropriate functional form. Regardless of the parametrization of system, the resulted optimization problem (sometimes nonconvex) is usually difficult to deal with, especially the global convergence of the estimated parameters. The use of Narendra-Gallman algorithm for the identification ${ }^{5,6}$ allows the prediction error to be separately linear in each set of parameters that characterize the linear and nonlinear parts. But it requires iterative procedures and could lead to divergent results in some cases. To develop noniterative algorithms, particular basis functions have been used to represent the static nonlinearity, ${ }^{7,8}$ and a linear regression problem for a parameter matrix containing product of the model parameters can be formulated. This parameter matrix is estimated with least-squares estimation, and the model parameters are then calculated by singular value decomposition of the parameter matrix. Although the optimization problem was simplified, parametrization of static nonlinearity is still required. Recently, Lee et al. ${ }^{9}$ proposed a method using a special test signal that enables decoupling the dynamic linear part and the static nonlinear part

* To whom correspondence should be addressed. E-mail: huanghpc@ ntu.edu.tw. Tel: 886-2-2363-8999. Fax: 886-2-2362-3935. for identification. But, it is applicable only to the case where nonlinearities are in terms of polynomial.

Motivated by the difficulties encountered in identifying an MIMO Hammerstein model, a new nonparametric method is presented in this paper. Parametrization of systems is not required, and complicated optimization problems are avoided. By using special test signals designed for this purpose, the identification of multivariable linear subsystem can be carried out under a SISO framework and can be decoupled from that of the static nonlinear function. As mentioned, due to the twopart structure of the process, models for input-output realization may not be unique; but they are all in equivalence. Among them, the one that has best linear dynamic characteristics could be used to develop the model predictive control for the process. In this paper, the identification as well as its application to model predictive control (MPC) will be illustrated.

\section{Problem Formulation}

A Hammerstein system is schematically represented as the one in Figure 1. The system consists of a static nonlinear function $\mathbf{F}(\bullet)$ followed by a linear time-invariant (LTI) dynamic element $\mathbf{G}(q)$. Let $\mathbf{H}(l) \in \mathbb{R}^{m \times n}$ with elements $\left\{h_{i j}(l)\right\}_{i=1,2, \ldots, n, j=1,2, \ldots, m}$ be the FIR matrix of $\mathbf{G}(q)$ and $\mathbf{F}(\bullet): \mathbb{R}^{m} \rightarrow \mathbb{R}^{m}$ be a nonlinear vector functional mapping. The Hammerstein representation of the process is then given by

$$
\begin{aligned}
\mathbf{y}(k) & =\sum_{l=0}^{L-1} \mathbf{H}(l) \mathbf{v}(k-l)+\mathbf{e}(k) \\
\mathbf{v}(k) & =\mathbf{F}(\mathbf{u}(k))
\end{aligned}
$$

where $\mathbf{u}(k), \mathbf{v}(k) \in \mathbb{R}^{m}, \mathbf{y}(k)$, and $\mathbf{e}(k) \in \mathbb{R}^{n}$, are the system input, intermediate input, output, and noise, respectively. The constant $L$ is the longest length among all of the impulse sequences, $h_{i j}(l)$. Based on a series of input $\mathbf{u}(k)$ and output $\mathbf{y}(k)$, the identification problem is formulated as the one to find $\mathbf{H}(l)$ and $\mathbf{F}(\bullet)$ that can predict $\hat{\mathbf{y}}(k)$, from $\mathbf{u}(k)$, with least sum of squared errors to the

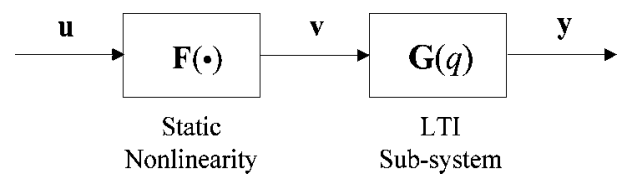

Figure 1. Structure of the Hammerstein model. 


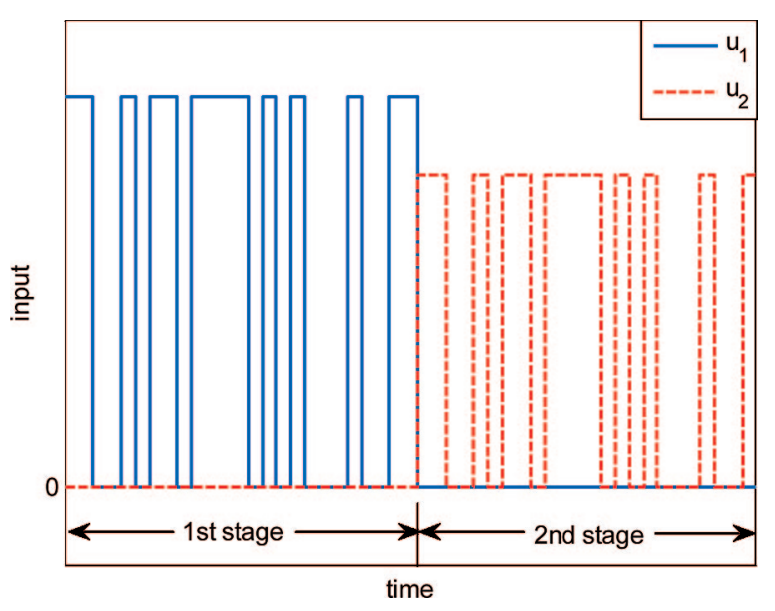

Figure 2. Inputs for the identification of the LTI subsystem.

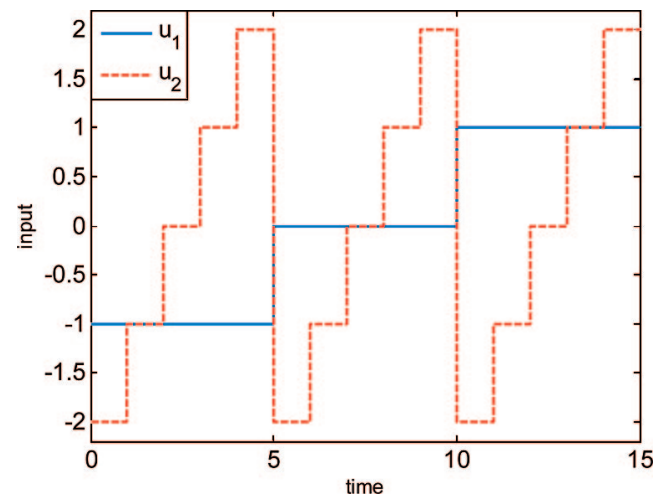

Figure 3. Inputs for the identification of static nonlinearity.

observation $\mathbf{y}(k)$. In this paper, the inputs are specially designed so as to enable sequentially estimations of $\mathbf{H}(l)$ and $\mathbf{F}(\bullet)$. No parametrization for both the functional nonlinearity and the LTI element is required at this stage. The only assumptions needed are that the LTI element is stable and the nonlinear function has a value of zero at the origin, i.e., $\mathbf{F}(\mathbf{0})=\mathbf{0}$.

For simplicity, formulation is based on a $2 \times 2$ system. However, extension to a general $m \times m$ system is straightforward without difficulty.

\section{Identification of LTI Subsystem}

If input and output data of a LTI linear MIMO system are available, a FIR matrix can be found using the linear least-squares (LS) method. ${ }^{10}$ Nevertheless, in such a Hammerstein system, input (i.e., $\mathbf{v}(k))$ to the linear subsystem is dummy and not measurable. But, if the static nonlinear function is driven by a two-level signal switching between zero and a nonzero constant, the resulting $\mathbf{v}(k)$ will be a two-level signal between zero and another nonzero constant. By this way, the LS method can be applied to estimate the FIR sequence. For this reason, a specially designed test signal to excite the linear subsystem is thus required.

3.1. Process Excitation. The test signal is generated by modulating each input with multistage inputs. In each stage, say stage $J, u_{J}(k)$ is a pseudorandom binary signal (PRBS) which switches between zero and a nonzero constant in a random manner while keeping all other inputs being zero (i.e., $u_{j}(k)=$ $0, j \neq J)$. The excitation signals are as shown in Figure 2. The number of such excitations equals the number of input variables. It follows that each dummy input $\left(v_{j}(k), j=1,2, \ldots, m\right)$ at the $J$ th stage is a PRBS switching between zero and a nonzero constant as well. Notice that each $v_{j}(k)$ is synchronized to $u_{J}(k)$.

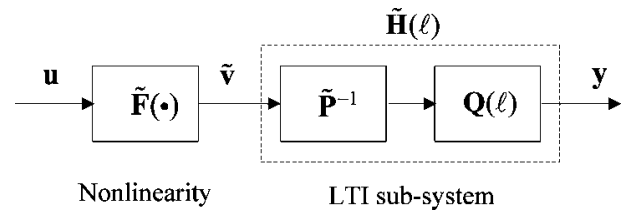

Figure 4. Realization model of the Hammerstein system.

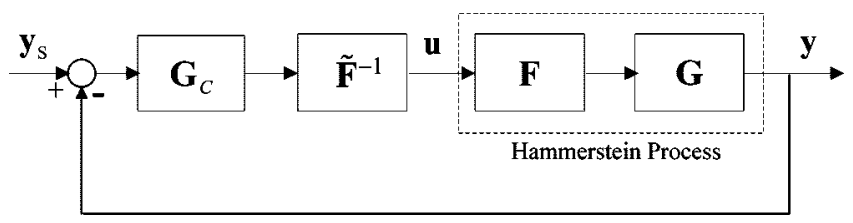

Figure 5. Control structure of the Hammerstein system.
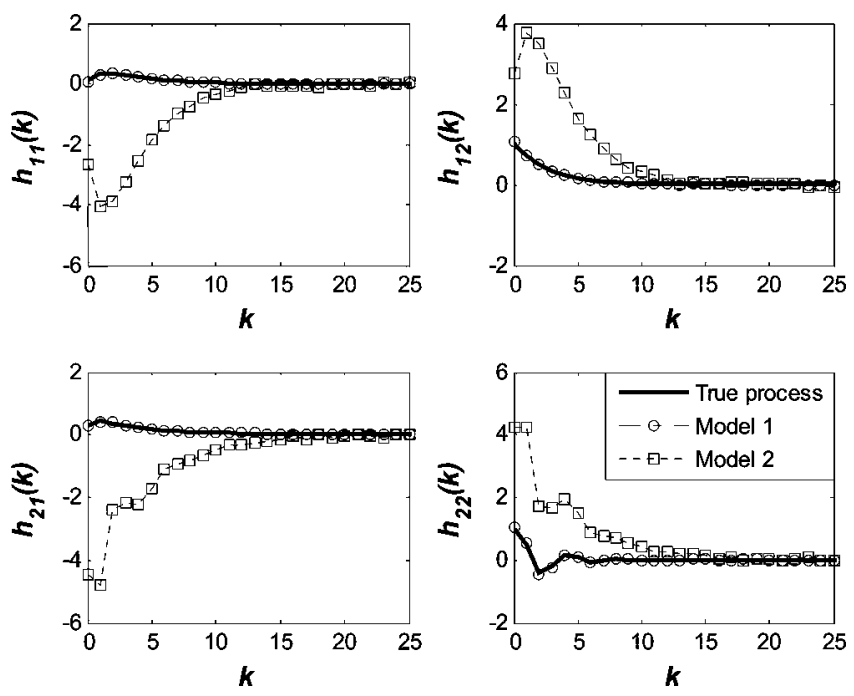

Figure 6. Impulse response of LTI subsystem in example 1.

As we will show in the following, such a test signal makes the identification of LTI element possible.

3.2. Estimation of FIR Matrix. Consider the LTI element in a $2 \times 2$ Hammerstein process. We denote a PRBS input that has levels at $a$ and $b$ as $\operatorname{PRBS}(a, b)$. The inputs at the first stage for excitation are $u_{1}(k)=\operatorname{PRBS}\left(\bar{u}_{1}, 0\right)$ and $u_{2}(k)=0$. As a result, the intermediate inputs at this stage are

$$
\begin{aligned}
& v_{1}(k)=\operatorname{PRBS}\left(f_{1}\left(\bar{u}_{1}, 0\right), 0\right) \\
& v_{2}(k)=\operatorname{PRBS}\left(f_{2}\left(\bar{u}_{1}, 0\right), 0\right)
\end{aligned}
$$

Then, at the second stage, $u_{1}(k)=0, u_{2}(k)=\operatorname{PRBS}\left(\bar{u}_{2}, 0\right)$ and thus we have

$$
\begin{aligned}
& v_{1}(k)=\operatorname{PRBS}\left(f_{1}\left(0, \bar{u}_{2}\right), 0\right) \\
& v_{2}(k)=\operatorname{PRBS}\left(f_{2}\left(0, \bar{u}_{2}\right), 0\right)
\end{aligned}
$$

The switch times of $v_{1}(k)$ and $v_{2}(k)$ is always synchronized to those of $u_{1}(k)$ and $u_{2}(k)$ in each of the two stages, respectively. The input-output relationship of eq 1 can be written, provided $\alpha_{1}, \alpha_{2} \neq 0$, as

$$
\begin{aligned}
\mathbf{y}(k) & =\sum_{l=0}^{L-1}\left[\begin{array}{ll}
h_{11}(l) & h_{21}(l) \\
h_{12}(l) & h_{22}(l)
\end{array}\right]\left[\begin{array}{l}
f_{1}(\mathbf{u}(k-l)) \\
f_{2}(\mathbf{u}(k-l))
\end{array}\right] \\
& =\sum_{l=0}^{L-1}\left[\begin{array}{cc}
\frac{h_{11}(l)}{\alpha_{1}} & \frac{h_{21}(l)}{\alpha_{2}} \\
\frac{h_{12}(l)}{\alpha_{1}} & \frac{h_{22}(l)}{\alpha_{2}}
\end{array}\right]\left[\begin{array}{l}
\alpha_{1} f_{1}(\mathbf{u}(k-l)) \\
\alpha_{2} f_{2}(\mathbf{u}(k-l))
\end{array}\right]
\end{aligned}
$$


Equation 4 shows that the nonlinear functions in $\mathbf{F}(\bullet)$ can be scaled by any nonzero constants because the LTI subsystem can compensate it accordingly. Thus, we can designate $f_{1}\left(\bar{u}_{1}, 0\right)$ as $\bar{u}_{1}$ and $f_{2}\left(0, \bar{u}_{2}\right)$ as $\bar{u}_{2}$, which means $\alpha_{1}=\bar{u}_{1} / f_{1}\left(\bar{u}_{1}, 0\right)$ and $\alpha_{2}=$ $\bar{u}_{2} / f_{2}\left(0, \bar{u}_{2}\right)$. Consequently, the dummy inputs at these two stages are defined as follows. At the first stage, $v_{1}(k)=u_{1}(k)$ and $v_{2}(k)$ $=p_{2} u_{1}(k)$, where $p_{2}=\alpha_{2} f_{2}\left(\bar{u}_{1}, 0\right) / \bar{u}_{1}$. Similarly, at the second stage, $v_{1}(k)=p_{1} u_{2}(k)$ and $v_{2}(k)=u_{2}(k)$, where $p_{1}=\alpha_{1} f_{1}\left(0, \bar{u}_{2}\right) /$ $\bar{u}_{2}$. Notice that the values of $\bar{u}_{1}$ and $\bar{u}_{2}$ are given by the excitation signals, while the values of $p_{1}$ and $p_{2}$ are parameters to be specified.

A $2 \times 2$ input-output realization of the LTI element in eq 1) can be written as
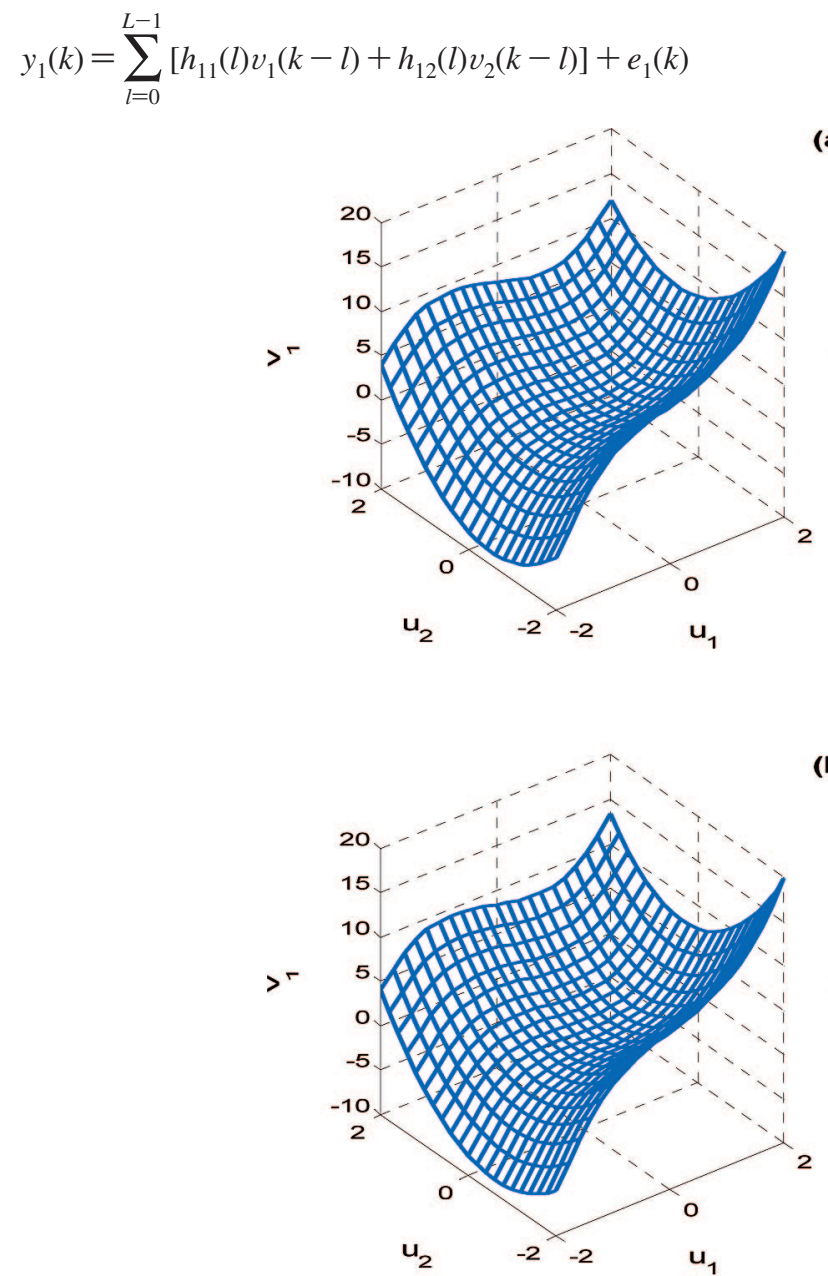

(b)

\section{(a)}

$y_{2}(k)=\sum_{l=0}^{L-1}\left[h_{21}(l)+p_{2} h_{22}(l)\right] u_{1}(k-l)+e_{2}(k)=$

$$
\sum_{l=0}^{L-1} h_{2}^{(1)}(l) u_{1}(k-l)+e_{2}(k)
$$
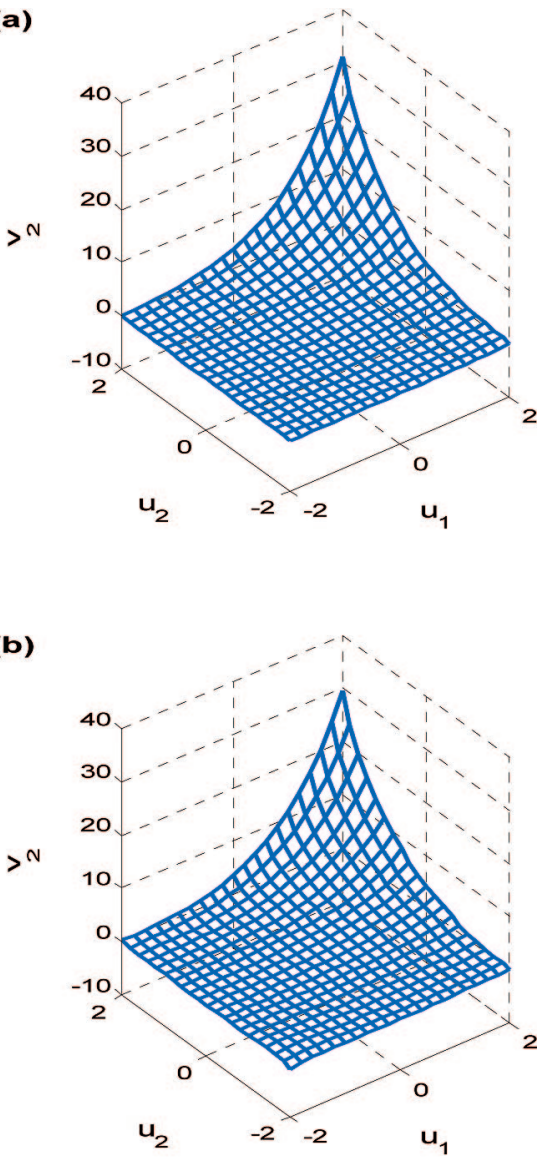
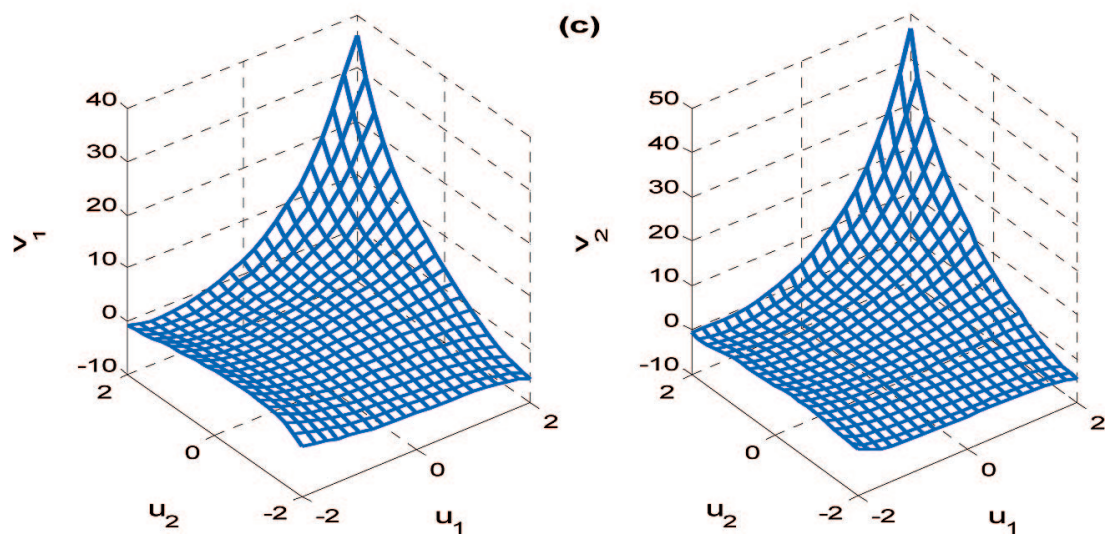

Figure 7. Plot of nonlinearity in example 1: (a) true process; (b) model 1; (c) model 2. 
And, in the second stage

$$
\begin{aligned}
& y_{1}(k)=\sum_{l=0}^{L-1}\left[p_{1} h_{11}(l)+h_{12}(l)\right] u_{2}(k-l)+e_{1}(k) \\
&=\sum_{l=0}^{L-1} h_{1}^{(2)} u_{2}(k-l)+e_{1}(k) \\
& y_{2}(k)=\sum_{l=0}^{L-1}\left[p_{1} h_{21}(l)+h_{22}(l)\right] u_{2}(k-l)+e_{2}(k) \\
&=\sum_{l=0}^{L-1} h_{2}^{(2)} u_{2}(k-l)+e_{2}(k)
\end{aligned}
$$

where

$$
\begin{aligned}
& h_{1}^{(1)}(l)=h_{11}(l)+p_{2} h_{12}(l) \\
& h_{2}^{(1)}(l)=h_{21}(l)+p_{2} h_{22}(l) \\
& h_{1}^{(2)}(l)=p_{1} h_{11}(l)+h_{12}(l) \\
& h_{2}^{(2)}(l)=p_{1} h_{21}(l)+h_{22}(l)
\end{aligned}
$$

With eq $6, h_{1}^{(1)}(l)$ and $h_{2}^{(1)}(l)$, for $l \in[0,1, \ldots, L-1]$, can be obtained from a conventional SISO least-squares estimation ${ }^{10}$ with available data from $\left(u_{1}, y_{1}\right)$ and $\left(u_{1}, y_{2}\right)$ at the first stage. Similarly, with eq $7, h_{1}^{(2)}(l)$ and $h_{2}^{(2)}(l)$ can be thus obtained with known data from $\left(u_{2}, y_{1}\right)$ and $\left(u_{2}, y_{2}\right)$ at the second stage. Rewrite eq 8 in matrix form as

$$
\mathbf{Q}(l)=\mathbf{H}(l) \mathbf{P}
$$

where

$$
\begin{aligned}
\mathbf{Q}(l) & =\left[\begin{array}{ll}
h_{1}^{(1)}(l) & h_{1}^{(2)}(l) \\
h_{2}^{(1)}(l) & h_{2}^{(2)}(l)
\end{array}\right] \\
\mathbf{H}(l) & =\left[\begin{array}{ll}
h_{11}(l) & h_{12}(l) \\
h_{21}(l) & h_{22}(l)
\end{array}\right] \\
\mathbf{P} & =\left[\begin{array}{ll}
1 & p_{1} \\
p_{2} & 1
\end{array}\right]
\end{aligned}
$$

So

$$
\mathbf{H}(l)=\mathbf{Q}(l) \mathbf{P}^{-1}, \quad l=0,1, \ldots, L-1
$$

Since the elements in $\mathbf{Q}(l)$ are estimated, the impulse response matrix $\mathbf{H}(l)$ can be computed by eq 11 , provided that values of $p_{1}$ and $p_{2}$ are given. Notice that the product of $p_{1}$ and $p_{2}$ must not equal to 1 (i.e., the matrix $\mathbf{P}$ cannot be singular).

Remark. A practical issue for FIR identification is the number of data that should be used to arrive at an accurate model. PRBS has a pulse-like autocorrelation function which closely approximates the delta function from an ideal white noise. Thus, least-squares identification using PRBS can result in a model with similar accuracy to that resulted from white noise excitation. Furthermore, if the data number $(N)$ is an integer multiple of the period of the PRBS, then the pulse-like autocorrelation function still results, even when the number of data points is relatively small. ${ }^{11}$ According to our experience in this study, $N$ is chosen as an integer multiple of the period of the PRBS with $N>5 L$, where $L$ is the length of FIR to be estimated.

\section{Identification of Nonlinearity}

With a set of given values of $p_{1}$ and $p_{2}$, (denoted as $\tilde{p}_{1}$ and $\tilde{p}_{2}$ ), the FIR matrix of the LTI subsystem is estimated by eq 11 as

$$
\widetilde{\mathbf{H}}(l)=\mathbf{Q}(l) \widetilde{\mathbf{P}}^{-1}
$$

The obtained $\tilde{\mathbf{H}}(l)$ and output $\mathbf{y}(k)$ can then be used to compute the unobserved intermediate variable, $\tilde{\mathbf{v}}(k)$, that corresponds to the given $\mathbf{u}(k)$. With a sufficient number of such correspondence $\mathbf{u}(k)$ and $\tilde{\mathbf{v}}(k)$ data pairs, it will be able to establish a nonlinear functional mapping between the two variables by interpolation.

4.1. Excitation for Static Nonlinearity. In order to define the nonlinear functional relationship between $\mathbf{u}(k)$ and $\tilde{\mathbf{v}}(k)$, the above-mentioned $\mathbf{u}(k)$ must cover the whole possible space of input variables. In addition, higher density of data pairs has to be used in the region where high model accuracy is desired. If there is not enough a priori knowledge about the process, signal with uniform density in the whole region is recommended. For this purpose, if the numbers of levels of $u_{1}$ and $u_{2}$ for excitation are $n_{1}$ and $n_{2}$, respectively, then the test signal contains a total of $n_{1} \times n_{2}$ pairs which are all the combinations of values of $u_{1}$ and of $u_{2}$. Notice that each pair lasts for one sampling interval only. For example, $u_{1}=\{-1,0,1\}$ and $u_{2}=\{-2,-1,0,1,2\}$, then there are 15 pairs in the test signal as shown in Figure 3. The sequence of these steps can be randomly ordered.

4.2. Estimation of Nonlinear Mapping. Assume the introduced multistep signal contains $n+1$ steps, i.e., $\mathbf{u}(k), k=0$, $1, \ldots, n$, and $\mathbf{u}(k)=\mathbf{0}$ for $k>n$. Then, based on $\tilde{\mathbf{H}}(l)$ and $\tilde{\mathbf{v}}(k)$, the LTI subsystem of eq 5 can be rewritten in a matrix form:

$$
\boldsymbol{\eta}=\widetilde{\boldsymbol{\Phi}} \widetilde{v}+\mathbf{e}
$$

where

$$
\widetilde{\boldsymbol{\Phi}}=\left[\begin{array}{ll}
\widetilde{\boldsymbol{\Phi}}_{11} & \widetilde{\boldsymbol{\Phi}_{12}} \\
\widetilde{\boldsymbol{\Phi}_{21}} & \widetilde{\boldsymbol{\Phi}}_{22}
\end{array}\right], \quad \widetilde{\boldsymbol{v}}=\left[\begin{array}{c}
\widetilde{\boldsymbol{v}}_{1} \\
\widetilde{\boldsymbol{v}}_{2}
\end{array}\right], \quad \boldsymbol{\eta}=\left[\begin{array}{l}
\boldsymbol{\eta}_{1} \\
\boldsymbol{\eta}_{2}
\end{array}\right], \quad \mathbf{e}=\left[\begin{array}{l}
\mathbf{e}_{1} \\
\mathbf{e}_{2}
\end{array}\right]
$$

with

$$
\begin{aligned}
\widetilde{\boldsymbol{\Phi}}_{i j} & =\left[\begin{array}{llll}
\widetilde{h}_{i j}(0) & 0 & \cdots & 0 \\
\tilde{h}_{i j}(1) & \tilde{h}_{i j}(0) & \cdots & 0 \\
\vdots & \vdots & \ddots & \vdots \\
\widetilde{h}_{i j}(n) & \tilde{h}_{i j}(n-1) & \cdots & \widetilde{h}_{i j}(0) \\
\vdots & \vdots & \ddots & \vdots \\
\tilde{h}_{i j}(n+L-1) & \tilde{h}_{i j}(n+L-2) & \cdots & \widetilde{h}_{i j}(L-1)
\end{array}\right], \widetilde{h}_{i j}(l)=0 \text { for } l \geq L \\
\widetilde{\boldsymbol{v}}_{j} & =\left[\begin{array}{llll}
\widetilde{v}_{j}(0) & \widetilde{v}_{j}(1) & \cdots & \widetilde{v}_{j}(n)
\end{array}\right]^{T} \\
\boldsymbol{\eta}_{i} & =\left[\begin{array}{llll}
y_{i}(0) & y_{i}(1) & \cdots & y_{i}(n+L-1)
\end{array}\right]^{T} \\
\mathbf{e}_{i} & =\left[\begin{array}{llll}
e_{i}(0) & e_{i}(1) & \cdots & e_{i}(n+L-1)
\end{array}\right]^{T}
\end{aligned}
$$

Notice that if the LTI element has time delay, the all-zero rows in $\tilde{\boldsymbol{\Phi}}$ and $\boldsymbol{\eta}$ have to be removed. Since the row number of $\tilde{\boldsymbol{\Phi}}$ is always larger than the column number and all elements of $\tilde{\boldsymbol{\Phi}}$ are identified, we can now estimate the unobserved intermediate variable $\tilde{\boldsymbol{v}}$ by the method of least-squares, i.e.

$$
\tilde{\boldsymbol{v}}=\left(\tilde{\boldsymbol{\Phi}}^{T} \tilde{\boldsymbol{\Phi}}\right)^{-1} \tilde{\boldsymbol{\Phi}}^{T} \boldsymbol{\eta}
$$

Thus, the nonlinearity is identified as the mapping from $\mathbf{u}(k)$ to $\tilde{\mathbf{v}}(k)$ thus obtained, that is, $\tilde{\mathbf{F}}(\bullet): \mathbf{u}(k) \rightarrow \hat{\tilde{v}}(k)$. Notice that we

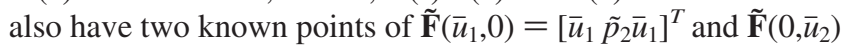
$=\left[\begin{array}{ll}\tilde{p}_{1} \bar{u}_{2} & \bar{u}_{2}\end{array}\right]^{T}$.

Having the FIR sequence and the nonlinear function, it will be able to predict the output from the given input, $\mathbf{u}(k)$. The model up to this stage has been identified. In general, this nonlinear function may be represented by an artificial neural network for interpolations. Or, it can be modeled as a multivariable polynomial with cross terms. For example, consider 
the nonlinear mapping to be represented by multivariable polynomials with order $M$ as the following:

$$
\hat{\tilde{v}}_{i}=\hat{f_{i}}\left(u_{1}, u_{2} ; \boldsymbol{\theta}_{i}\right)=\sum_{j=0}^{M} \theta_{i, j} u_{1}^{M-j} u_{2}^{j}
$$

The parameters can be estimated by regression

$$
\theta_{i}^{*}=\arg \min _{\theta_{i}} \sum_{k=0}^{n}\left\{\hat{f}_{i}\left(u_{1}(k), u_{2}(k) ; \boldsymbol{\theta}_{i}\right)-\hat{\tilde{v}}_{i}(k)\right\}^{2}
$$

Remarks. According to eq $13, n+L$ data points are used for the identification of nonlinearity. The selection of $n$ may depend on the complexity of the nonlinearity, the range of the operating region, and the desired model accuracy.

4.3. Measurement Noise. If the noise $\mathbf{e}$ is random and white, $\hat{\tilde{v}}$ in eq 15 is an unbiased estimate of $\tilde{\boldsymbol{v}}$. Moreover, if the number of rows in $\tilde{\boldsymbol{\Phi}}$ approaches infinity, $\hat{\tilde{v}}$ is a consistent estimate of $\tilde{\boldsymbol{v}}$. To increase the row number of $\tilde{\boldsymbol{\Phi}}$, we can proceed to test using the signal of which the steps are identical to those of previously used input, but the sequence is randomly scrambled. In this way, more equations for the unknown $\tilde{\boldsymbol{v}}$ can be set up in eq 13 and hence a more consistent estimate of $\tilde{\boldsymbol{v}}$ could result. However, this is at the cost of prolonged experiment time. In case of strong noise level, it is suggested that the measurement is first passed through a filter to reduce the effect of noise and maintain accurate identification of model without extra test. Another way to deal with the noise is fitting the estimated data set with a nonlinear function because the noise will be filtered out by the fitting procedure.

\section{Tuning Matrix $\tilde{\mathbf{P}}$ for Model-Based Control}

According to the identification algorithm presented in sections 3 and 4, the resulting Hammerstein model is given in Figure 4 in a nonparametric form. In this model, the LTI element consists of two blocks, $\mathbf{Q}(l)$ and $\tilde{\mathbf{P}}^{-1}$, where $\mathbf{Q}(l)$ is defined by several identified SISO FIR sequences. Based on the given $\tilde{\mathbf{P}}$, the associated intermediate variable $\tilde{\mathbf{v}}$ is estimated and then a nonlinearity $\tilde{\mathbf{F}}(\bullet)$ results accordingly. Generally, the values of $\tilde{p}_{1}$ and $\tilde{p}_{2}$ can be arbitrarily given (provided $\tilde{p}_{1} \tilde{p}_{2} \neq 1$ ), so that these two components of a MIMO Hammerstein model are not unique. In other words, for a given input-output set, many combinations of different static nonlinearity $\tilde{\mathbf{F}}(\bullet)$ and LTI subsystem $\tilde{\mathbf{H}}(l)$ may have identical outputs. Therefore, it gives engineers the flexibility to choose a model representation for better control. In the proposed model shown in Figure 4, this flexibility is achieved by selecting the values at the off-diagonal entries of $\tilde{\mathbf{P}}$. By a proper selection of this $\tilde{\mathbf{P}}$ matrix, parametric models are developed for model-based control uses.

A control structure for Hammerstein type nonlinear process is as shown in Figure 5, where $\mathbf{G}_{C}$ can be a conventional PID controller or an MPC controller. ${ }^{12}$ Assume the nonlinearity $\tilde{\mathbf{F}}(\bullet)$ is invertible so that the design of $\mathbf{G}_{C}$ depends only on $\tilde{\mathbf{H}}(l)$. Thus, the off-diagonal elements of $\tilde{\mathbf{P}}$ can be determined to meet the desired characteristic imposed on the LTI subsystem $\tilde{\mathbf{H}}(l)$. Two cases with different purposes of design are discussed in the following:

Steady-State Decoupling. If the system is square, the matrix $\tilde{\mathbf{P}}^{-1}$ can be aimed to serve as a steady-state decoupler for $\mathbf{Q}(l)$, i.e., the LTI subsystem $\tilde{\mathbf{H}}(l)$ is "self-decoupled" at steady state. Let $K_{l}^{(j)}=\sum_{l=0}^{L-1} h_{l}^{(j)}(l)$ be the steady-state gain of the dynamics described by $h_{l}^{(j)}(l)$ and $\mathbf{K}$ be the steady-state gain matrix of $\mathbf{Q}(l)$ in eq 10 with

$$
\mathbf{K}=\left[\begin{array}{cc}
K_{1}^{(1)} & K_{1}^{(2)} \\
K_{2}^{(1)} & K_{2}^{(2)}
\end{array}\right]
$$

By eq 12 , if $\tilde{\mathbf{P}}$ is chosen as $\tilde{\mathbf{P}}_{\mathrm{d}}$ such that $\mathbf{K} \tilde{\mathbf{P}}_{\mathrm{d}}{ }^{-1}$ is a diagonal matrix, the linear subsystem described by $\tilde{\mathbf{H}}(l)$ is decoupled at steady state. In other words, a steady-state decoupler, $\tilde{\mathbf{P}}_{\mathrm{d}}^{-1}$, has been automatically embedded into the model. Let $\mathbf{D}=\mathbf{K} \tilde{\mathbf{P}}_{\mathrm{d}}{ }^{-1}$.
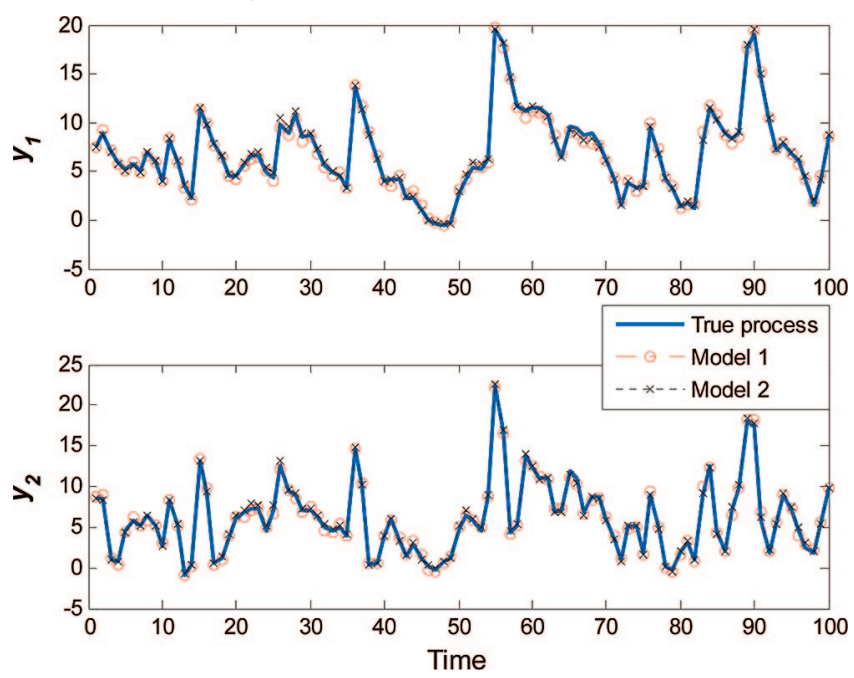

Figure 8. Process and model outputs to random input in example 1
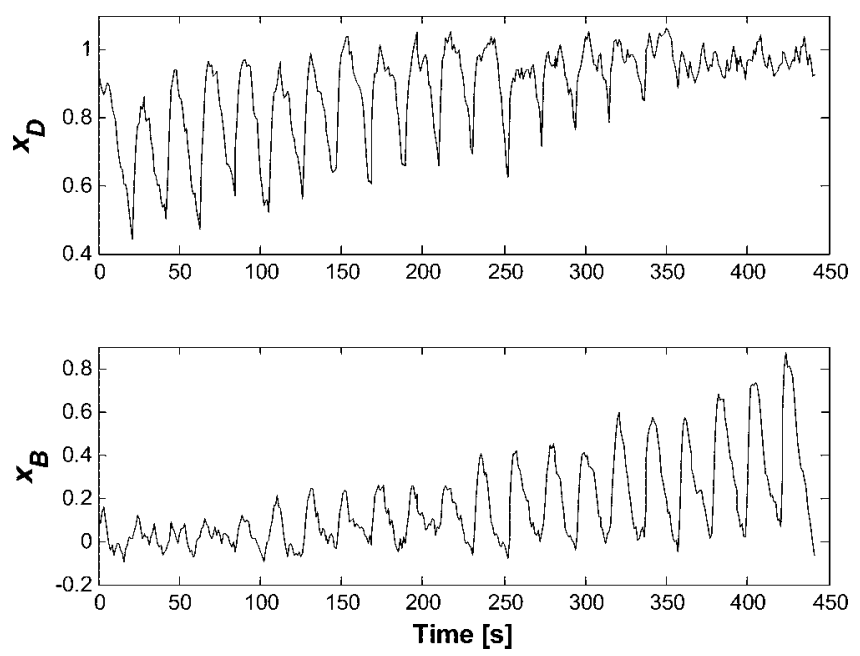

Figure 9. Output response to multistep inputs in example 2.
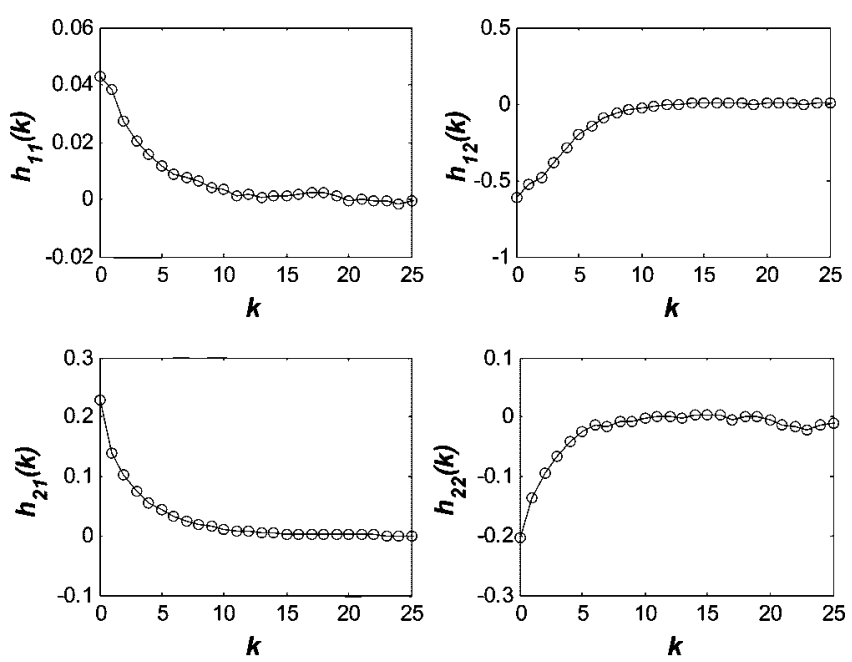

Figure 10. Impulse response of LTI subsystem in example 2. 

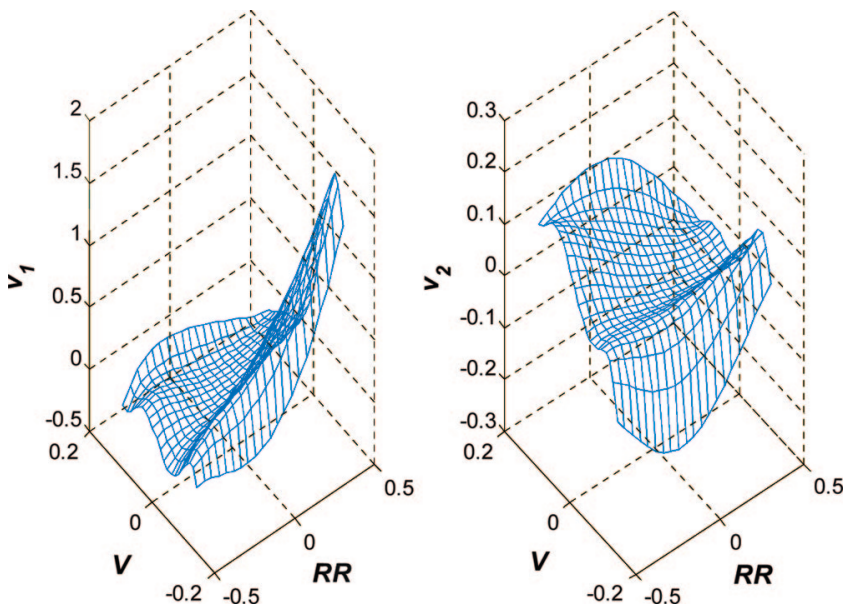

Figure 11. Plot of nonlinearity in example 2.
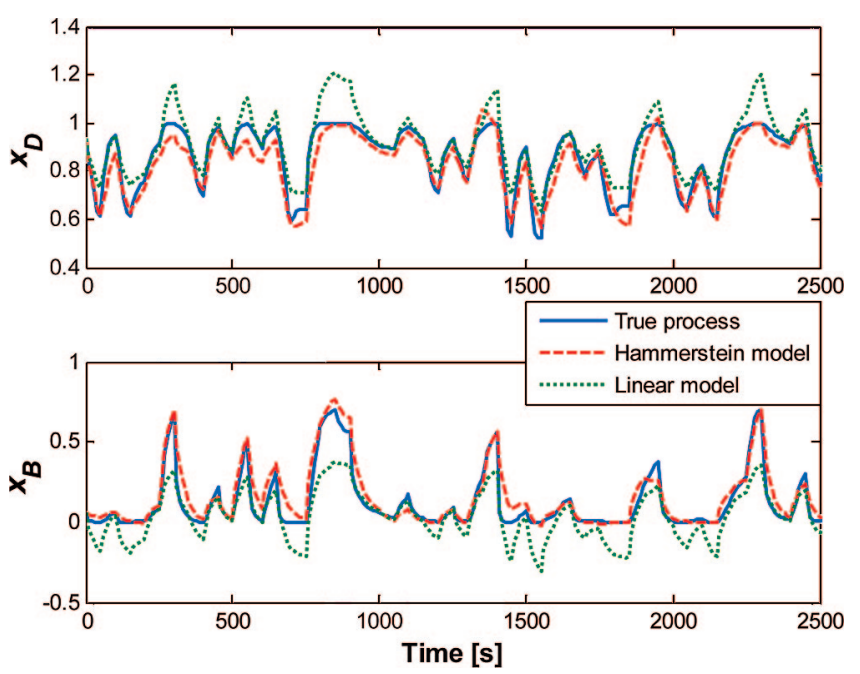

Figure 12. Process and model outputs to random input in example 2.

The off-diagonal elements of $\tilde{\mathbf{P}}_{\mathrm{d}}$ are obtained by solving the equation

$$
\left\{D_{i j}\right\}_{i=1,2, \ldots, n ; j=1,2, \ldots, m ; i \neq j}=0
$$

For a $2 \times 2$ system, the resulting $\tilde{\mathbf{P}}_{\mathrm{d}}$ is

$$
\widetilde{\mathbf{P}_{\mathrm{d}}}=\left[\begin{array}{ll}
1 & K_{2}^{(1)} / K_{2}^{(2)} \\
K_{1}^{(2)} / K_{1}^{(1)} & 1
\end{array}\right]
$$

Diagonal Dominance. The diagonal dominance of a MIMO model is usually desired because it means slight interactions between loops, so that the design of multiloop decentralized controller would be easier. To this end, the off-diagonal elements of $\tilde{\mathbf{P}}$ can be estimated by solving the following optimization problem to reduce the loop interactions

$$
\left\{\tilde{p}_{1}^{*}, \tilde{p}_{2}^{*}\right\}=\arg \min _{\widetilde{p_{1}}, \tilde{p}_{2}}\left(w_{1} \frac{\left\|\tilde{\mathbf{h}}_{12}\right\|_{2}}{\left\|\tilde{\mathbf{h}}_{11}\right\|_{2}}+w_{2} \frac{\left\|\tilde{\mathbf{h}}_{21}\right\|_{2}}{\left\|\tilde{\mathbf{h}}_{22}\right\|_{2}}\right)
$$

where $w_{1}$ and $w_{2}$ are weighting factors of two loops and $\tilde{\mathbf{h}}_{i j}=$ $\left[\tilde{h}_{i j}(0) \tilde{h}_{i j}(1) \cdots \tilde{h}_{i j}(L-1)\right]^{T}$. Alternatively, similar to steadystate decoupling, $\tilde{\mathbf{P}}$ can also be estimated to maximize the diagonal dominance of the LTI model at a certain frequency $\omega$ by letting

$$
\widetilde{\mathbf{P}}_{\mathrm{dd}}=\left[\begin{array}{ll}
1 & K_{2}^{(1)}(\omega) / K_{2}^{(2)}(\omega) \\
K_{1}^{(2)}(\omega) / K_{1}^{(1)}(\omega) & 1
\end{array}\right]
$$

where

$$
K_{i}^{(j)}(\omega)=\left|\sum_{l=0}^{L_{l-1}} h_{i}^{(j)}(l) \mathrm{e}^{-j l \omega}\right|
$$

Remark. As mentioned, the nonparametric input-output realization model of Hammerstein process is not unique. The model can be made unique if a priori knowledge about the LTI element in terms of transfer function matrix is incorporated. For example, the linear elements in the LTI subsystem is of ARX form with known order $\left(r_{i j}, s_{i j}\right)$ and delay $d_{i j}$, i.e.

$$
\begin{aligned}
G_{i j}(q) & =\frac{y_{i}(t)}{v_{j}(t)}=\frac{B_{i j}(q) q^{-d_{i j}}}{A_{i j}(q)} \\
& =\frac{\left(b_{i j, 0}+b_{i j, 1} q^{-1}+b_{i j, 2} q^{-2}+\cdots+b_{i j, s_{i j}} q^{-s_{i j}}\right) q^{-d_{i j}}}{1-a_{i j, 1} q^{-1}-a_{i j, 2} q^{-2}-\cdots-a_{i j, r_{i j}} q^{-r_{i j}}}
\end{aligned}
$$

The corresponding FIR sequence, for $l>\left(d_{i j}+s_{i j}\right)$ satisfies the following relation:

$$
h_{i j}(l)=a_{i j, 1} h_{i j}(l-1)+a_{i j, 2} h_{i j}(l-2)+\cdots+a_{i j, r_{i j}} h_{i j}\left(l-r_{i j}\right)
$$

Thus, the off-diagonal elements of $\tilde{\mathbf{P}}$ can be determined such that the condition in eq 24 is satisfied. Although the parameters $a_{i j}$ are unknown, they can be computed from $\tilde{h}_{i j}(l)$ by the method of least-squares, if the off-diagonal elements of $\tilde{\mathbf{P}}$ are given. That is

$$
\mathbf{a}_{i j}=\left(\boldsymbol{\Gamma}_{i j}^{T} \boldsymbol{\Gamma}_{i j}\right)^{-1} \boldsymbol{\Gamma}_{i j}^{T} \boldsymbol{\varphi}_{i j}
$$

where

$$
\begin{aligned}
\mathbf{a}_{i j} & =\left[\begin{array}{llll}
a_{i j, 1} & a_{i j, 2} & \cdots & a_{i j, r_{i j}}
\end{array}\right]^{T} \\
\boldsymbol{\varphi}_{i j} & =\left[\begin{array}{llll}
\tilde{h}_{i j}\left(d_{i j}+s_{i j}+1\right) & \tilde{h}_{i j}\left(d_{i j}+s_{i j}+2\right) & \cdots & \tilde{h}_{i j}(L)
\end{array}\right]^{T} \\
\boldsymbol{\Gamma}_{i j} & =\left[\begin{array}{llll}
\tilde{h}_{i j}\left(d_{i j}+s_{i j}\right) & \tilde{h}_{i j}\left(d_{i j}+s_{i j}-1\right) & \cdots & \tilde{h}_{i j}\left(d_{i j}+s_{i j}-r_{i j}+1\right) \\
\tilde{h}_{i j}\left(d_{i j}+s_{i j}+1\right) & \tilde{h}_{i j}\left(d_{i j}+s_{i j}\right) & \cdots & \tilde{h}_{i j}\left(d_{i j}+s_{i j}-r_{i j}+2\right) \\
\vdots & \vdots & \ddots & \vdots \\
\tilde{h}_{i j}(L-1) & \tilde{h}_{i j}(L-2) & \cdots & \tilde{h}_{i j}\left(L-r_{i j}\right)
\end{array}\right]
\end{aligned}
$$

Notice that $\boldsymbol{\varphi}_{i j}, \boldsymbol{\Gamma}_{i j}$, and $\mathbf{a}_{i j}$ are functions of the off-diagonal elements of $\tilde{\mathbf{P}}$. Thus, these off-diagonal elements can be determined by solving an optimization problem. For example, a $2 \times 2$ system, it is

$$
\left\{\widetilde{p}_{1}^{*}, \widetilde{p}_{2}^{*}\right\}=\arg \min _{\widetilde{p_{1}}, \widetilde{p_{1}}}\left(\sum_{i=1}^{2} \sum_{j=1}^{2}\left\|\boldsymbol{\varphi}_{i j}-\boldsymbol{\Gamma}_{i j} \mathbf{a}_{i j}\right\|_{2}\right)
$$

As a result, a unique representation of the Hammerstein model is obtained. If the model order and delay are unknown, the above optimization problem can be solved repetitively using different sets of $\left(r_{i j}, s_{i j}, d_{i j}\right)$ until the residual is smaller than required.

\section{Simulation Example}

Example 1. Consider a nonlinear process described by the Hammerstein system as follows:

$$
\begin{aligned}
& \mathbf{G}(q)=\left[\begin{array}{ll}
\frac{0.1 q^{-1}+0.2 q^{-2}}{1-1.2 q^{-1}+0.35 q^{-2}} & \frac{q^{-1}}{1-0.7 q^{-1}} \\
\frac{0.3 q^{-1}+0.2 q^{-2}}{1-0.8 q^{-1}} & \frac{q^{-1}+0.5 q^{-2}}{1+0.4 q^{-2}}
\end{array}\right] \\
& \mathbf{F}(\mathbf{u})=\left[\begin{array}{l}
u_{1}^{3}-u_{1} u_{2}+2 u_{2}^{2} \\
0.582\left(\mathrm{e}^{\left(u_{1}+u_{2}\right)}-1\right)
\end{array}\right]
\end{aligned}
$$


(a)

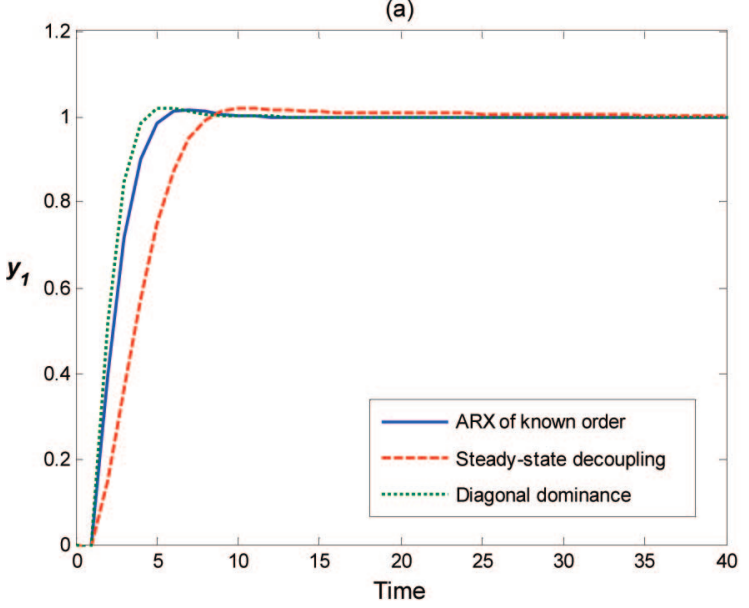

(c)

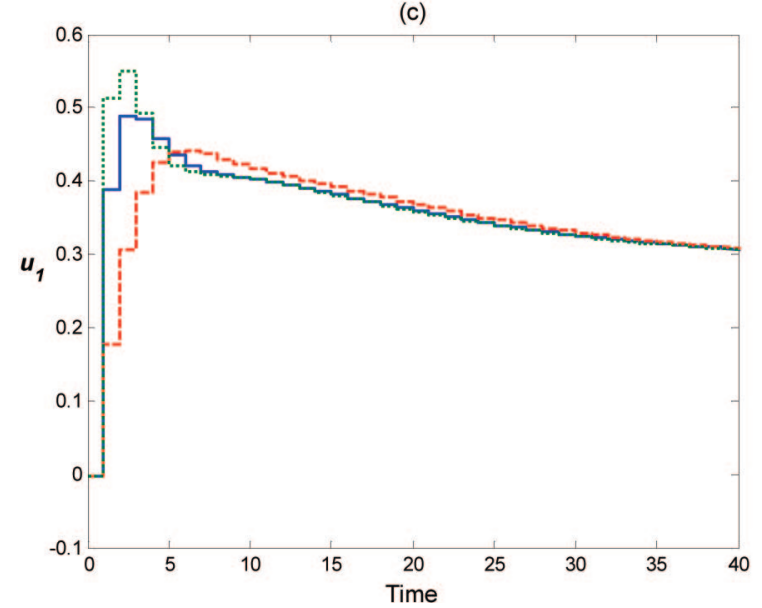

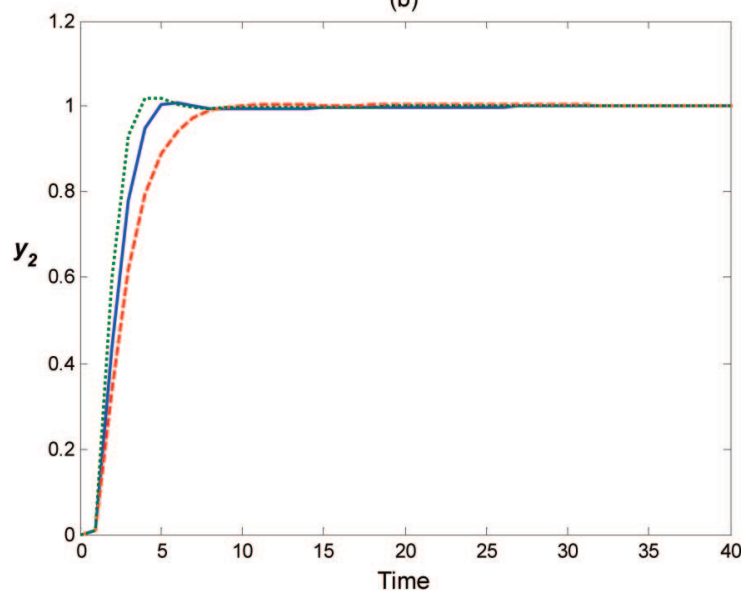

(d)

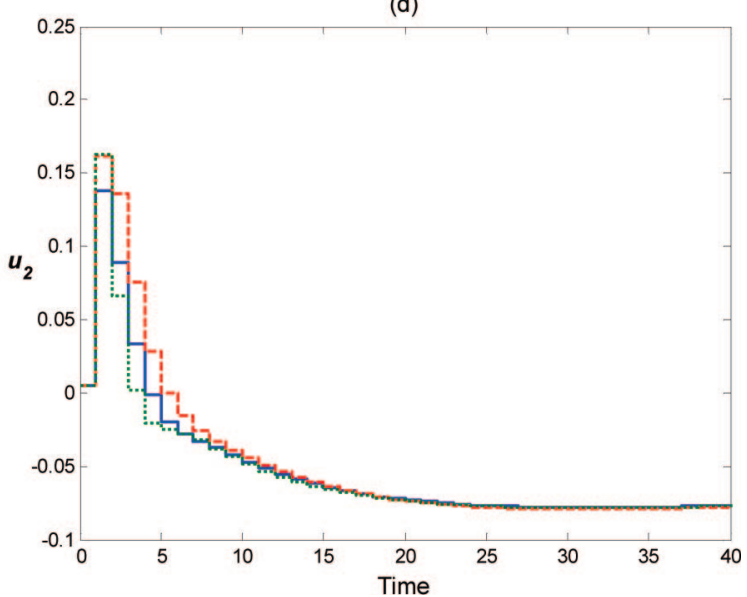

Figure 13. Nonlinear MPC results in example 3.

The inputs for estimation of FIR matrix are chosen as $u_{1}(k)$ $=\operatorname{PRBS}(1,0), u_{2}(k)=0$ at the first stage and $u_{1}(k)=0, u_{2}(k)$ $=\operatorname{PRBS}(1,0)$ at the second stage. The input for identification of the nonlinearity is a multivariable multistep signal which covers all the combination of $u_{1}=u_{2}=\{-2: 0.2: 2\}$, i.e., a total of $21 \times 21=441$ pairs. The sampling interval is taken as 1 . To simulate the measurement noise, random white noise is added to the output, with $\left|e_{1}(k)\right| \leq 0.2$ and $\left|e_{2}(k)\right| \leq 0.2$. First, the matrix $\mathbf{Q}(l)$ is identified from the PRBS test. Here, two models with different characteristics for the LTI subsystem are used for illustration. First, the order $\left(r_{i j}, s_{i j}\right)$ of ARX dynamics for the LTI subsystem is assumed known (model 1). The results obtained by eq 26 are $\tilde{p}_{1}=1.973$ and $\tilde{p}_{2}=0.965$. Notice that their exact values are $p_{1}=2$ and $p_{2}=1$. In the second one, steady-state decoupling is imposed on the LTI subsystem (model 2 ), and the results computed from eq 20 are $\tilde{p}_{1}=0.592$ and $\tilde{p}_{2}$ $=1.357$. The impulse response sequences of these two models together with that of original process are shown in Figure 6. Based on the two LTI models, two corresponding input-output mappings of the nonlinearity are thus estimated from the test of multistep signal. These mappings have been fitted with multivariable polynomials as shown in Figure 7. The outputs of original Hammerstein system and two identified models to random input are simulated as shown in Figure 8. As mentioned previously, although these two representations of the Hammerstein model are quite different, both of them can produce very similar outputs to that of the original system.
Example 2. Consider a distillation column with 30 trays for the separation of a binary mixture as it was used by Benallou et al. ${ }^{13}$ and Horton et al. ${ }^{14}$ The column is described by 32 nonlinear ordinary differential equations and is assumed to have a constant molar overflow as well as a constant relative volatility of 1.6. The feed stream is introduced at the middle of the column on stage 17 and has a composition of $x_{\mathrm{F}}=0.5$. The reflux ratio (RR) and the vapor boilup $(V)$ are the input variables $\left(u_{1}\right.$ and $\left.u_{2}\right)$, and the distillate composition $\left(x_{\mathrm{D}}\right)$ and bottom composition $\left(x_{\mathrm{B}}\right)$ are used as output variables $\left(y_{1}\right.$ and $\left.y_{2}\right)$. The steady-state values of these four variables are $\mathrm{RR}=3, V=0.8, x_{\mathrm{D}}=0.935$, and $x_{\mathrm{B}}=0.065$.

The proposed method is used to identify a Hammerstein model based on simulation data with a sampling time of $10 \mathrm{~s}$. The operating region for this system was chosen to be $\pm 15 \%$ around the steady-state vales. The exciting signals for the estimation of FIR matrix are $u_{1}(k)=\operatorname{PRBS}(0.225,0), u_{2}(k)=0$ at the first stage and $u_{1}(k)=0, u_{2}(k)=\operatorname{PRBS}(0.06,0)$ at the second stage. Then, a multivariable multistep signal which covers all the combination of $u_{1}=\{-0.45: 0.045: 0.45\}, u_{2}=$ $\{-0.12: 0.012: 0.12\}$ is used to estimate the nonlinearity. Colored noises, $w(k)$, with autoregressive dynamics of order 1 (i.e., $w(k)$ $=0.8 w(k-1)+e(k))$ are added to the outputs to simulate a more realistic scenario. The noise-to-signal ratio (NSR), defined as the following, is around $20 \%$.

$$
\mathrm{NSR}=\frac{\text { mean }(\text { abs }(\text { noise }))}{\text { mean }(\text { abs }(\text { signal }))}
$$


The output response to multistep inputs is shown in Figure 9. Based on the excitation of PRBS, the matrix $\mathbf{Q}(l)$ is first identified. We assume that all dynamics of the LTI subsystem are represented as ARX of order $(1,1)$ without time delay, and the parameters are found as $\tilde{p}_{1}=-0.213$ and $\tilde{p}_{2}=-0.014$. The identified impulse response sequences are shown in Figure 10. Based on this LTI model, the corresponding input-output mappings of the nonlinearity are thus estimated from the test of multistep signal. This mapping has been fitted with multivariable polynomials of order 5, as shown in Figure 11. The simulated response of the identified Hammerstein model is compared with the actual response in Figure 12, where a good agreement between them can be observed. For the purpose of comparison, random signals covering the operating region were used to excite the process, and a linear model (FIR matrix) is thus identified using the least-squares estimation. The simulated response of the identified linear model is also shown in Figure 12. Clearly, the predictive capability of the Hammerstein model outperforms that of the linear model.

Example 3. To demonstrate that different model representations can be used for nonlinear control application, a Hammerstein process with simple nonlinearity as follows is considered.

$$
\begin{aligned}
\mathbf{G}(q) & =\left[\begin{array}{ll}
\frac{-0.112 q^{-1}-0.058 q^{-2}}{1-1.05 q^{-1}+0.136 q^{-2}} & \frac{0.59 q^{-1}}{1-0.607 q^{-1}} \\
\frac{0.85 q^{-1}}{1-0.717 q^{-1}} & \frac{0.098 q^{-1}}{1-0.95 q^{-1}}
\end{array}\right] \\
\mathbf{F}(\mathbf{u}) & =\left[\begin{array}{l}
u_{1}^{2}+2 u_{2} \\
2 u_{1}-u_{2}^{2}
\end{array}\right]
\end{aligned}
$$

The operating region is $u_{1}, u_{2} \in\left[\begin{array}{ll}-1 & 1\end{array}\right]$, and the sampling interval is 1 . Three identified Hammerstein models with different characteristics for the LTI subsystem (i.e., ARX of known order, steady-state decoupling, and diagonal dominance) are used for the nonlinear MPC design given in Figure 5. The control horizon and prediction horizon are taken as 2 and 5, respectively, in all simulations. Each input and output is equally weighted. The closed-loop responses are shown in Figure 13. It can be seen that all the identified models can be used for control design with satisfactory responses. Nevertheless, better control performance is found when the model with diagonally dominant dynamics for the LTI subsystem is used.

\section{Conclusion}

In this paper, a new method has been presented to identify and model MIMO Hammerstein systems. By the proposed special test inputs, the identifications of LTI subsystem and nonlinearity are separated, so that iterative procedures are avoided. Because the proposed method is a nonparametric one, the parametrization of system is not required in advance and thus the representation of model is not unique. With the identified nonparametric realization of the system, engineers can have the flexibility in modeling Hammerstein systems to meet their demands by adjusting a few parameters. Simulation results have shown that an accurate model can be identified by the proposed method. Furthermore, different model representations can be used to describe the behavior of a Hammerstein system and can be used for control design.

Note Added after ASAP Publication: The version of this paper that was published on the Web July 30, 2008 has errors in it that were associated with eq 2 . The corrected version of this paper was reposted to the Web August 5, 2008.

\section{Literature Cited}

(1) Verhaegen, M.; Westwick, D. Identifying MIMO Hammerstein Systems in the Context of Sub-Space Model Identification Methods. Int. J. Control 1996, 63, 331-349.

(2) Al-Duwaish; Karim, M. N. A New Method for the Identification of Hammerstein Model. Automatica 1997, 33, 1871-1875.

(3) Patwardhan, R. S.; Lakshminarayanan, S.; Shah, S. L. Constrained Nonlinear MPC Using Hammerstein and Wiener Models: PLS Framework. AIChE J. 1998, 44, 1611-1622.

(4) Rollins, D. K.; Bhandari, N.; Bassily, A. M.; Colver, G. M.; Chin, S. T. A. Continuous-Time Nonlinear Dynamic Predictive Modeling Method for Hammerstein Processes. Ind. Eng. Chem. Res. 2003, 42, 860-872.

(5) Lakshminarayanan, S.; Shah, S. L.; Nandakumar, K. Identification of Hammerstein Models Using Multivariate Statistical Tools. Chem. Eng. Sci. 1995, 50, 3599-3613.

(6) Eskinat, E.; Johnson, S. H.; Luyben, W. L. Use of Hammerstein Models in Identification of Nonlinear Systems. AIChE J. 1991, 37, 255268.

(7) Gomez, J. C.; Baeyens, E. Identification of Block-Oriented Nonlinear Systems Using Orthonormal Bases. J. Process Control 2004, 14, 685-697.

(8) Chan, K. H.; Bao, J.; Whiten, W. J. Identification of MIMO Hammerstein Systems Using Cardinal Spline Functions. J. Process Control 2006, 16, 659-670.

(9) Lee, Y. J.; Sung, S. W.; Park, S.; Park, S. Input Test Signal Design and Parameter Estimation Method for the Hammerstein Wiener Processes. Ind. Eng. Chem. Res. 2004, 43, 7521-7530.

(10) Hsia, T. C. System Identification: Least-Squares Methods; D.C. Heath and Co.: Lexington, MA, 1977.

(11) Sung, S. W.; Lee, J. H. Pseudo.Random Binary Sequence Design for Finite Impulse Response Identification. Control Eng. Practice 2003, $11,935-947$.

(12) Fruzzetti, K. P.; Palazoglu, A.; McDonald, K. A. Nonlinear Model Predictive Control Using Hammerstein Models. J. Process Control 1997, 7, 31-41.

(13) Benallou, A.; Seborg, D. E.; Mellichamp, D. A. Dynamic Compartmental Models for Separation Processes. AIChE J. 1986, 32, 10671078.

(14) Horton, R. R.; Bequette, B. W.; Edgar, T. F. Improvements in Dynamic Compartmental Modeling for Distillation. Comput. Chem. Eng. 1991, 15, 197-201.

Received for review November 7, 2007 Revised manuscript received May 15, 2008 Accepted May 23, 2008

IE071512Q 